\title{
EFICÁCIA DE SOLUÇÕES DESINFETANTES NA ELIMINAÇÃO DE FUNGOS DE IMPORTÂNCIA MÉDICA E VETERINÁRIA
}

\author{
Isabel Martins Madrid², Alessandra Jacomelli Teles ${ }^{1}$, Rosema Santin², Antonella \\ Souza Mattei ${ }^{2}$, Angelita Gomes ${ }^{1}$, Stefanie B Waller ${ }^{1}$ \\ 1 UFPel \\ 2 UFRGS \\ Correspondência: Isabel Martins Madrid: imadrid_rs@yahoo.com.br
}

RESUMO: Programas de higiene e desinfecção são essenciais no controle ambiental de agentes fúngicos potencialmente patogênicos aos homens e animais, dessa forma objetivou-se avaliar a ação antifúngica do hipoclorito de sódio $4 \%$ e do digluconato de clorexidina $6,6 \%$ em doze isolados fúngicos. Foram testados fungos isolados do ambiente e de casos clínicos como Aspergillus spp.. Candida spp.., Microsporum spp.., Malassezia pachydermatis, Cryptococcus neoformans e Sporothrix schenckii. Foram utilizadas as técnicas de microdiluição em caldo e difusão em ágar conforme protocolos descritos pelo Clinical and Laboratory Standards Institute (CLSI) com adaptações para agentes químicos. Todos os isolados foram sensíveis a concentrações inferiores a recomendada pelo fabricante do digluconato de clorexidina $(6,6 \%)$ com concentração inibitória mínima (CIM) e concentração fungicida mínima (CFM) entre $\leq 0,42$ a 1,68\%. Para o hipoclorito de sódio, $58,3 \%$ dos isolados foram resistentes à concentração recomendada pelo fabricante $(4 \%)$ apresentando CIM e CFM entre 2 e $>8 \%$. O digluconato de clorexidina apresentou maiores zonas de inibição do que o hipoclorito de sódio, com halos de até $31 \mathrm{~mm}$ de diâmetro. A clorexidina demonstrou ação fungicida em baixas concentrações sendo eficaz na eliminação de fungos filamentosos e leveduriformes de importância médica e veterinária, enquanto o hipoclorito de sódio somente obteve esta ação em altas concentrações.

Palavras-chave: hipoclorito de sódio; digluconato de clorexidina; leveduras; dermatófitos; Aspergillus spp..

\section{EFFICACY OF DISINFECTANT SOLUTIONS IN THE ELIMINATION OF FUNGI OF MEDICAL AND VETERINARY IMPORTANCE}

\begin{abstract}
Hygiene and disinfection programs are essential in the environmental control of fungal agents potentially pathogenic to humans and animals. In this study it was evaluated the antifungal activity of $4 \%$ sodium hypochlorite and $6.6 \%$ chlorexidine digluconate solutions against twelve fungal isolates. Fungi such as Aspergillus spp.., Candida spp.., Microsporum spp.., Malassezia pachydermatis, Cryptococcus neoformans and Sporothrix schenckii obtained from environment and clinical cases were tested. Broth microdilution and agar diffusion techniques describes by the Clinical and Laboratory Standard Institute (CLSI) adapted to the chemical solutions were utilized. All isolates were sensible in concentrations minor of the recommended by manufacture of the chlorexidine digluconate $(6.6 \%)$ with minimal inhibitory concentration (MIC) and minimal fungicidal concentration (MFC) $\leq 0.42$ to $1.68 \%$. To sodium hypochlorite was observed that $58.3 \%$ of the isolates were resistant to the concentration recommended by manufacturer (4\%) with MIC and MFC values between 2 and $>8 \%$. Chlorexidine digluconate presented greater inhibition zone than sodium hypochlorite, with halos of even $31 \mathrm{~mm}$ of diameter. Chlorexidine demonstrated fungicidal action in low concentrations being efficient in the elimination of mycelial fungi and yeasts of medical and veterinary importance, while the sodium hypochlorite only obtained this action in high concentrations.
\end{abstract} Key Words: sodium hypochlorite; chlorexidine digluconate; yeasts; dermatophytes; Aspergillus spp.. 


\section{INTRODUÇÃO}

O aumento de diagnósticos de doenças causadas por fungos nas últimas décadas alerta para a importância do controle destes agentes como causadores de infecção e contaminação de ambientes. Dados relativos a humanos demonstram um aumento de $207 \%$ na incidência de fungemias, entre os anos de 1979 e 2000, acometendo principalmente imunocomprometidos e pacientes hospitalizados com sérias doenças de base (Pfaller \& Diekema, 2007). Em medicina veterinária, os casos de infecções causadas por Candida spp.., Malassezia pachydermatis, Sporothrix schenckii e dermatófitos têm sido descritos com frequência em pequenos animais, assim como, infecções por Aspergillus spp.. em animais marinhos (Cleff et al., 2007; Xavier et al., 2007a; Madrid et al., 2010)

Os fungos possuem ampla distribuição na natureza, podendo ser encontrados no ar, água, terra, animais e alimentos, sendo responsáveis pela contaminação de diversos materiais. Em ambientes clínicos e hospitalares, a presença de fungos e bactérias no ar e superfícies é frequente (Alangaden, 2011; Mattei et al., 2011a). Recentemente, Mattei et al. (2011b) isolaram o fungo Sporothrix schenckii de ambiente hospitalar veterinário e domiciliar, alertando para a possibilidade de infecção de animais e humanos presentes nestes ambientes.

As infecções fúngicas são consideradas uma das causas do aumento da morbidade e mortalidade de pacientes hospitalizados. Evidências de transmissão de infecções por Candida spp.., Aspergillus spp.. e Cryptococcus neoformans pelo meio ambiente são bem descritas na literatura (Anvisa, 2010; Wang et al., 2005). Neste sentido, o processo de desinfecção torna-se de extrema importância podendo resultar na redução de $99 \%$ da carga microbiana, quando associado à limpeza prévia (Rutala \& Werber, 2004).

Programas de higiene e desinfecção são essenciais no controle ambiental de agentes potencialmente patogênicos aos homens e animais, e desse modo o uso de desinfetantes torna-se a ferramenta principal e um dos procedimentos fundamentais de biossegurança. Para tanto, o produto químico escolhido deve realizar, efetivamente, as funções de descontaminação e desinfecção (Bambace et al., 2003). A desinfecção ambiental deve ser realizada com rodízio periódico de agentes químicos capazes de eliminar ou inibir 0 crescimento microbiano (Xavier et al., 2007b).

Visto 0 potencial patogênico apresentado por muitos fungos torna-se importante a desinfecção correta do ambiente, assim, a escolha de um produto com amplo espectro de ação e com pouca interferência por matéria orgânica é fundamental para a eliminação fungos patogênicos do ambiente. Dessa forma, o presente estudo avaliou a ação antifúngica do hipoclorito de sódio e digluconato de clorexidina através das técnicas de difusão em ágar e microdiluição em caldo frente a fungos de importância médica e veterinária.

\section{MATERIAL E MÉTODOS}

A sensibilidade de doze isolados fúngicos foi avaliada frente a solução aquosa de desinfetantes com princípio ativo de hipoclorito de sódio $4 \%$ e digluconato de clorexidina $6,6 \%$ através das técnicas de microdiluição em caldo e difusão em ágar.

Para a realização dos testes de suscetibilidade foram utilizados isolados ambientais e clínicos de Aspergillus 
apresentou valores de CIM e CFM que variaram de $2 \%$ para os isolados de $M$. canis e $M$. gypseum, 4\% para $M$. pachydermatis $(\mathrm{n}=2)$, S. schenckii $(\mathrm{n}=1)$ e de $8 \%$ para os isolados $C$. guilliermondii, C. famata, C. neoformans $(\mathrm{n}=2)$, A. flavus, $A$. niger e $S$. schenckii $(\mathrm{n}=1)$. O hipoclorito de sódio apresentou CIM e CFM maior que a concentração indicada pelo fabricante do desinfetante (4\%) em $58,3 \%$ (7/12) dos isolados testados.

No teste de disco difusão os maiores halos de inibição observados foram do digluconato de clorexidina que alcançaram 30 e $31 \mathrm{~mm}$ de diâmetro frente aos isolados de $M$. pachydermatis, seguido de $S$. schenckii com 18 e $17 \mathrm{~mm}, M$. canis e M. gypseum com 13 e $12 \mathrm{~mm}, C$. neoformans com 6 e $8 \mathrm{~mm}$, C. guilliermondii e C. famata com $7 \mathrm{~mm}$ e $A$. niger com $5 \mathrm{~mm}$, não sendo observada zona de inibição para A. flavus. Já para o desinfetante a base de hipoclorito de sódio a ausência de zona de inibição do crescimento foi observada em 83,3\% (10/12) dos isolados, estando presente apenas frente os isolados $C$. guilliermondii (8mm) e C. famata $(7 \mathrm{~mm})$.

\section{DISCUSSÃO}

Em medicina veterinária, pesquisas a respeito da atividade antifúngica de agentes químicos utilizados na desinfecção de ambientes veterinários são escassos (Santos et al., 2007; Xavier et al., 2007b). Entretanto, estudos acerca da atividade antifúngica da clorexidina e do hipoclorito de sódio têm sido realizados extensivamente na área da odontologia (Waltimo et al., 1999; Estrela et al., 2003; Fraiser et al., 2004; Vianna et al., 2004; Menezes et al., 2008; Chandra et al., 2010).

A ação antimicrobiana da clorexidina tem demonstrado resultados contraditórios, sendo considerado que sua ação frente a fungos é menos eficaz do que em bactérias (Fraiser et al., 2004). Entretanto, pesquisas relacionadas ao potencial fungicida contra leveduras, especialmente do gênero Candida, mostram que a clorexidina é eficaz, agindo em concentrações de 0,2 a 2\% (Vianna et al., 2004; Chandra et al., 2010). Guardabassi et al. (2010) demonstraram maior sensibilidade de Malassezia spp. à clorexidina quando comparada com bactérias como Proteus mirabilis, Staphylococcus aureus, Escherichia coli e Pseudomonas aeruginosa.

Os resultados encontrados no presente estudo corroboram os dados descritos por outros autores, demonstrando que concentrações em torno de $0,5 \%$ de clorexidina são eficazes em fungos leveduriformes e filamentosos como $C$. albicans, $C$. neoformans, $A$. fumigatus, $A$. niger e $A$. flavus (Bambace et al., 2003; Théraud et al., 2004; Xavier et al., 2007b). Na difusão em ágar, a clorexidina apresentou variações no tamanho do halo de inibição de acordo com a espécie fúngica testada com halos inferiores a $10 \mathrm{~mm}$ para isolados de Aspergillus spp., Candida spp. e Cryptococcus neoformans e, superiores a $10 \mathrm{~mm}$ para Malassezia pachydermatis, Microsporum spp. e Sporothrix schenckii. Diferentemente, Estrela et al. (2003) demonstraram halos de inibição de $16 \mathrm{~mm}$ utilizando isolados de Candida albicans.

O hipoclorito de sódio é indicado para desinfecção de superfícies não metálicas e com presença de matéria orgânica, apresentando efeito fungicida em concentrações de 0,02 a 1\% (Brasil, 2010). A atividade antibacteriana do hipoclorito de sódio $1 \%$ também foi demonstrada frente a bactérias de importância na avicultura como Salmonella enteritidis, Staphylococcus aureus e Escherichia coli (Jaenisch et 
al., 2010). A ação fungistática e fungicida do hipoclorito e produtos a base de cloro em concentrações menor ou igual a $1 \%$ foi descrita em $C$. neoformans, $C$. albicans e espécies de Trichophyton (Waltimo et al., 1999; Gupta et al., 2001; Théraud et al., 2004; Wang et al., 2005). Entretanto, outros autores demonstraram que esta ação foi obtida somente em concentrações de 2 e $5,25 \%$ para $A$. niger e $C$. albicans, respectivamente (Vianna et al., 2004; Pap et al., 2006). Porém, deve-se considerar que as técnicas utilizadas no presente estudo para avaliar a ação antifúngica do hipoclorito de sódio diferem das utilizadas pelos autores acima descritos, 0 que pode ter determinado a baixa eficácia do hipoclorito de sódio encontrada neste estudo.

Os valores de CIM e CFM obtidos pela técnica de microdiluição se assemelham aos descritos por Menezes et al. (2008) sendo menores para clorexidina do que para o hipoclorito de sódio. De forma similar, Estrela et al. (2003) também verificaram menor tamanho de halo de inibição na técnica de difusão para hipoclorito de sódio do que para clorexidina.

No presente estudo não foram observadas diferenças na suscetibilidade entre fungos filamentosos e leveduriformes frente aos agentes químicos testados embora, alguns autores descrevam que fungos filamentosos são menos sensíveis a agentes biocidas e desinfetantes do que leveduras (Fraiser et al., 2004). Ambas as técnicas utilizadas apresentaram resultados similares, sendo que pela microdiluição em caldo foi possível determinar a CIM e CFM enquanto, que a difusão em ágar apenas determinou presença ou ausência de crescimento fúngico, considerando que não existem valores de halos de inibição protocolados, de forma a determinar a sensibilidade do micro-organismo em sensível, intermediário ou resistente ao produto testado.

\section{CONCLUSÃO}

O digluconato de clorexidina e o hipoclorito de sódio demonstraram ação fungicida com resultados similares entre as técnicas testadas. $\mathrm{O}$ digluconato de clorexidina apresentou atividade em baixas concentrações sendo eficaz na eliminação de fungos filamentosos e leveduriformes de importância médica e veterinária, enquanto o hipoclorito de sódio somente obteve esta ação em altas concentrações.

\section{AGRADECIMENTOS}

Os autores agradecem ao Conselho Nacional de Desenvolvimento Científico e Tecnológico (CNPq) e a Fundação de Amparo à Pesquisa do Estado do Rio Grande do Sul (FAPERGS) pelo auxílio financeiro.

\section{REFERÊNCIAS}

ALANGADEN, G.J. Nosocomial fungal infections: epidemiology, infection control and prevention. Infectious Disease Clinics of North America, v.25, n.1, p.201-225, 2011.

AGÊNCIA NACIONAL DE VIGILÂNCIA SANITÁRIA - ANVISA. Segurança do paciente em serviços de saúde: Limpeza e desinfecção de superfície. Brasília: Anvisa, 2010. 116p.

BAMBACE, A.M.J.; BARROS, É.J.A.; SANTOS, S.S.F. et al. Eficácia das soluções aquosas de clorexidina para desinfecção de superfícies. Revista Biociências, v.9 (Supl. 2), p.73-81, 2003.

CHANDRA, S.S. ; MIGLANI, R.; SRINIVASAN, M.R . et al. Antifungal Efficacy of $5.25 \%$ Sodium Hypochlorite, 2\% Chlorhexidine Gluconate, and $17 \%$ EDTA With and Without an Antifungal Agent. Journal of Endodontics, v.36, n.4, p.675-678, 2010.

CLEFF, M.B.; SILVA, G.M.; MEINERZ, A.R.M.; et al. Infecção cutânea em cão por Candida albicans. Veterinária e Zootecnia, v.14, n.2, p.164-168, 2007. 
ESTRELA, C.; RIBEIRO, R.G.; ESTRELA, C.R.A. et al. Antimicrobial effect of $2 \%$ sodium hypochlorite and $2 \%$ chlorhexidine tested by different methods. Brazilian Dental Journal, v.14, n.1, p.58-62, 2003.

FRAISE, A.P.; LAMBERT, P.A.; MAILLARD, J-Y (Eds.). Principles and Practice of Disinfection, Preservation \& Sterilization. 4. ed. Oxford, Blackwell Inc.; 2004. 678p.

GUARDABASSI, L.; GHIBAUDO, G.;

DAMBORG, P. In vitro antimicrobial activity of a commercial ear antiseptic containing chlorhexidine and Tris-EDTA. Veterinary

Dermatology, v.21, p.282-286, 2009.

GUPTA, A.K.; AHMAD, I.; SUMMERBELL, R.C. Comparative efficacies of commonly used disinfectants and antifungal pharmaceutical spray preparations against dermatophytic fungi. Medical Mycology, v.39, p.321-328, 2001.

JAENISCH, F.R.F. ; KUCHIISHI, S.S. ; COLDEBELLA, A. Atividade antibacteriana de desinfetantes para uso na produção orgânica de aves. Ciência Rural, v.40, n.2, p.384-388, 2010.

MADRID, I.M.; MATTEI, A.; MARTINS, A. et al. Feline Sporotrichosis in the Southern Region of Rio Grande Do Sul, Brazil: Clinical, Zoonotic and Therapeutic Aspects. Zoonoses and Public Health, v.57, n.2, p.151-154, 2010.

MATTEI, A.S. ; SANTIN, R.; MADRID, I.M. et al. Isolamento de fungos filamentosos e gênero Staphylococcus em locais de atendimento veterinário. A Hora Veterinária, v.30, n.180, p.10-22, 2011 a.

MATTEI, A.S.; MADRID, I.M.; SANTIN, R. et al. Sporothrix schenckii in a hospital and home environment in the city of Pelotas/RS - Brazil.

Anais da Academia Brasileira de Ciências, v.83, n.4, p.1359-1362, 2011b.

MENEZES, M.M.; OLIVEIRA, L.D.; KOGA-ITO, C.Y. et al. Concentração fungicida mínima das soluções de clorexidina e hipoclorito de sódio sobre Candida albicans. Ciências

Odontológicas Brasileira, v.11, n.2, p.23-28, 2008.

PAP, K.; SZILLI, M.; KISKÓ, G. Testing antimicrobial efficiency of seven disinfectants against bacteria and fungi with surface test. Acta Alimentaria, v.35, p.163-170, 2006.

PFALLER, M.A.; DIEKEMA, D.J. Epidemiology of Invasive Candidiasis: a Persistent Public Health Problem. Clinical Microbiology

Reviews, v.20, n.1, p.133-163, 2007.
RUTALA, W.A.; WERBER, D.J. The benefits of surface disinfection. American Journal of Infection Control, v.32, n.4, p.226-231, 2004.

SANTOS, L.R.; NETO, J.F.S.; RIZZO, N.N. et al. Eficácia de desinfetantes e anti-sépticos empregados no hospital veterinário da UPF (HVUPF) Brasil. Revista da FZVA, v.14, n.2, p.156164, 2007.

THÉRAUD, M.; BÉDOUIN, Y.; GUIGUEN, C. et al. Efficacy of antiseptics and disinfectants on clinical and environmental yeast isolates in planktonic and biofilm conditions. Journal of Medical Microbiology, v.53, p.1013-1018, 2004.

VIANNA, M.E.; GOMES, B.P.; BERBER, V.B. et al. In vitro evaluation of the antimicrobial activity of chlorhexidine and sodium hypochlorite. Oral Surgery, Oral Medicine, Oral Pathology, Oral Radiology and Endodontology, v.97, n.1, p.79-84, 2004.

WALTIMO, T.M.; ORSTA,V.I.K.; SIRÉN, E.K. et al. In vitro susceptibility of Candida albicans to four disinfectants and their combinations. International Endodontic Journal, v.32, n.6, p.421-429, 1999.

WANG, C.Y.; WU, H.D.; HSUEH, P.R. Nosocomial transmission of cryptococcosis. The New England Journal of Medicine, v.352, n.12, p.1271-1272, 2005.

XAVIER, M.O.; SOARES, M.P.; MEINERZ, A.R.M.; et al. Aspergillosis: a limiting factor during recovery of captive Magellanic Penguins. Brazilian Journal of Microbiology, v.38, n.3, p.480-484, 2007a.

XAVIER, M.O.; MADRID, I.M.; MEIRNERZ, A.R.M.; et al. Atividade in vitro de três agentes químicos frente a diferentes espécies de Aspergillus. Arquivos do Instituto Biológico, v.74, n.1, p.49-53, 2007b. 